\title{
Cyclic Growth and Global Stability of Economic Dynamics of Kaldor Type in Two Dimensions
}

\author{
Aka Fulgence Nindjin, Albin Tetchi N'Guessan, Hypolithe Okou, and Kessé Thiban Tia
}

UFR de Mathématiques et Informatique, Université Félix Houphouët Boigny d’Abidjan Cocody, 22 BP 582,

Abidjan 22, Côte D'Ivoire

Correspondence should be addressed to Hypolithe Okou; akpetihi.okou@univ-fhb.edu.ci

Received 6 January 2017; Accepted 2 May 2017; Published 2 July 2017

Academic Editor: Patricia J. Y. Wong

Copyright (C) 2017 Aka Fulgence Nindjin et al. This is an open access article distributed under the Creative Commons Attribution License, which permits unrestricted use, distribution, and reproduction in any medium, provided the original work is properly cited.

\begin{abstract}
This article proposes nonlinear economic dynamics continuous in two dimensions of Kaldor type, the saving rate and the investment rate, which are functions of ecological origin verifying the nonwasting properties of the resources and economic assumption of Kaldor. The important results of this study contain the notions of bounded solutions, the existence of an attractive set, local and global stability of equilibrium, the system permanence, and the existence of a limit cycle.
\end{abstract}

\section{Introduction}

The nonlinear complex dynamics have been introduced in the analysis of the economic phenomena to explain on the one hand the fluctuations noticed in the study of the chronological series and on the other hand the economic crisis in the capitalist system. The economists Goodwin (1967) and Kaldor (1955-1956) used the dynamic samples to explain that the graphs of cyclic and chaotic evolution are endogeneous to the economic system itself.

To simplify things, a great number of these models have been elaborated with more restrictive assumption such as linearity. The challenges of structural reforms of dynamics justify the fact that mathematicians are interested in them. Our contribution will thus consist in proposing economic models inspired by the ecological models whose lessons may be important in terms of analyzing systems and their regulation in this context of climate protection (cf. $[1,2])$.

The basic economic models that we use in this article are those of Kaldor proposed in the works of Hans-Walter Lorenz (cf. [3]).

Our study will consist first of modifying the models of Kaldor conferring them with the ecological properties adapted to economy. Therefore, we propose a dynamic model typical of Kaldor-Holling-2 and Leslie-Gower with some modifications. Next, we will study the qualitative comportment of the model at level 2. We set criteria for which we have on the one hand the marking out of the solutions and the existence of an attractive set and, on the overhand, the local stability of the equilibrium and the permanence of the system.

At last we study the global stability of one interior equilibrium through the construction of the Lyapunov function.

\section{Dynamics of Kaldor Ecologic in Two Dimensions}

2.1. Economic Dynamics of Type Kaldor with the Effective Growth Rate in Two Dimensions. Let us start by giving the notations and definitions of the rates of parameters and functions in applied economies.

(1) The productivity of capital is the quotient of the GDP $Y$ by the capital $K$. We set that $\sigma=Y / K$.

(2) The rate of investment is the quotient of the investment $I$ by the GDP. We note that $f=I / Y$.

(3) The saving rate of the GDP $\phi$ is the quotient of the saving $S$ by the GDP. We have $\phi=S / Y$.

(4) The rate accumulation of the capital is denoted by $h=$ $I / K=\sigma f$. 
(5) We design, respectively, by $\varrho$ and $\alpha$ the monetary depreciation rate and the monetary adjustment coefficient.

(6) The ratio saving-capital will be noted as $g=S / K=$ $\sigma \phi$. 44):

Let us consider the original model of Kaldor (cf. [3] page

$$
\begin{aligned}
\dot{Y}= & \alpha[I(Y, K)-S(Y, K)], \\
\dot{K}= & I(Y, K)-\varrho K, \\
& \varrho, \alpha, Y(0), K(0) \in \mathbb{R}_{+}, Y, K \in C^{1}\left(\left[0 ;+\infty\left[; \mathbb{R}_{+}\right) .\right.\right.
\end{aligned}
$$

In order to establish a connection between the economic models of Kaldor and the ecological patterns, let us give up the coercion of monetary scales by writing investment and saving functions in relation to the investment rate, of capital and saving hoarding. Let us also replace the growth rate of the GDP of model (1) by its effective growth rate (cf. [3, 4]). Therefore we get the following assumption.

Assumption 1 (Kaldor with effective growth). (1) Effective growth $\dot{Y} / Y=\operatorname{Trend}(Y)+\alpha((I-S) / Y)$ with the coefficient of monetary mending, $\alpha \geq 0$ and the tendency, Trend $(Y)$, such that $\operatorname{Trend}(0) \geq 0$.

(2) The ratio saving-capital, $g(Y, K)$, is a function verifying $\partial g(Y, K) / \partial Y>0$.

(3) The investment rate $f(Y, K)$ is a function verifying $\partial f(Y, K) / \partial Y>0$ and there is a threshold $K_{s} \geq 0$ so that $\partial f(Y, K) / \partial K<0, \forall K \geq K_{s}$.

(4) The accumulation rate of the capital $h(Y, K)$ is a function verifying $\partial h(Y, K) / \partial Y>0, \partial h(Y, K) / \partial K<0$.

With Assumption 1, we get below the dynamics of Kaldor with an effective growth rate whose $f(Y, K)$ and $g(Y, K)$ can have ecological properties.

$$
\begin{aligned}
\dot{Y}= & \operatorname{Trend}(Y) Y+\alpha f(Y, K) Y-\alpha g(Y, K) K, \\
\dot{K}= & h(Y, K) K-\varrho K, \\
& \varrho, \alpha, Y(0), K(0) \in \mathbb{R}_{+}, Y, K \in C^{1}\left(\left[0 ;+\infty\left[; \mathbb{R}_{+}\right) .\right.\right.
\end{aligned}
$$

Interpretation. The dynamics of Kaldor with an effective growth rate (2) present some similarities to the classical ecological dynamics. The function $g_{Y}(Y, K)=\alpha[f(Y, K)-$ $\phi(Y, K)] Y$ define the action of the capital $K$ upon production $Y$. It stimulates the increase of the production when the investment rate $f$ is superior to the saving rate $\phi$ and stops it in the end.

The function $g_{K}(Y, K)=h(Y, K) K$ defines in its part the action of the production over the capital. The economic model alone summarizes two types of ecological interaction such as mutualising type and the prey-predators type. Let us consider the economic assumption of ecological inspirations on investment rate $f$, the rate of capital accumulation $h$, and the ratio saving-capital $g$.

Assumption 2.

$$
\begin{aligned}
& f(Y, K)=\widetilde{b}_{1}(K)\left(1-\frac{m_{2} K}{Y+C_{2}}\right) \\
& \text { with } \widetilde{b}_{1}(K)=a_{1} K+b_{1}, \\
& h(Y, K)=\widetilde{b}_{2}(Y)\left(1-\frac{m_{2} K}{Y+C_{2}}\right) \\
& g(Y, K)=\frac{m_{1} Y}{Y+C_{1}},
\end{aligned}
$$

where $a_{1}, a_{2}, b_{1}, b_{2}, m_{1}, C_{1} \in \mathbb{R}$.

Obviously, the investments are first funded by capitals. We can thus suppose that $f(Y, K)=\widetilde{b}_{1}(K)>0$. Next, the investors adjust the rate in relation to the realities for they hate to invest in vain. However it is not necessary to invest more when net profit is beyond expectancies. Let us estimate the losses of the investment by using the function $L G(Y, K)=$ $m_{2} K /\left(Y+C_{2}\right)$, where $m_{2}$ is the maximum value of the losses of the investment rate and $C_{2}$ is the maximum value of the stock of capital. Then we get $f(Y, K)=\widetilde{b}_{1}(K)-L G(Y, K) \widetilde{b}_{1}(K)=$ $\widetilde{b}_{1}(K)\left(1-m_{2} K /\left(Y+C_{2}\right)\right)$. For simplicity, we can take $\widetilde{b}_{1}(K)=$ $a_{1} K+b_{1}$, where $a_{1}$ and $b_{1}$ are the constants depending on the economic policies of investments. Then, we get a rate of investment that verifies the economic requirements (5) of Assumption 1.

Concerning the accumulation of capital, it is known that $h(Y, K)=\sigma f(Y, K)=\widetilde{b}_{2}(Y)\left(1-m_{2} K /\left(Y+C_{2}\right)\right)$ so $\widetilde{b}_{2}(Y)=$ $a_{1} Y+\sigma b_{1}$. If $\sigma=\left(\left(a_{2}-a_{1}\right) Y+b_{2}\right) / b_{1}$ then $\tilde{b}_{2}(Y)=a_{2} Y+b_{2}$ with $a_{2}$ the part of GDP converted in the stock of capital and $b_{2} \in \mathbb{R}$. Therefore $h(Y, K)$ verifies the economic constraint (11) of Assumption 1.

Concerning the saving, let us take $S(Y, K)=g(Y) K$, where $g(Y)=m_{1} Y /\left(Y+C_{1}\right)$ is the ratio saving-capital. The function $g$ verifies condition (4) of Assumption 1.

Suppose now that the tendency is linear and decreasing: $\operatorname{Trend}(Y)=a_{0}\left(1-Y / C_{0}\right)$. In fact, the tendency of the growth rate of GDP is at the start, a constant $a_{0}$ for a given period (forthcoming). But it faces some losses due, for example, to corruption, bribes, slush funds, tax haven, whitening of fraudulent funds, manipulations of accounts and media, and any other harmful activity to the growth of the GDP (cf. [5], pages 11, 15-16, and 35-65). Those losses are estimated to $a_{0} Y / C_{0}$ with $C_{0}$ the maximum value (monetary) of the GDP that we can get from this economy for the given period. 
Then, model (2) becomes the economic dynamics:

$$
\begin{aligned}
\dot{Y}= & {\left[a_{0} Y\left(1-\frac{Y}{C_{0}}\right)\right]+\alpha\left(a_{1} K+b_{1}\right) Y\left(1-\frac{m_{2} K}{Y+C_{2}}\right) } \\
& -\left[\alpha \frac{m_{1} Y}{Y+C_{1}}\right] K, \\
\dot{K}= & {\left[\left(a_{2} Y+b_{2}\right)\left(1-\frac{m_{2} K}{Y+C_{2}}\right)\right] K-\varrho K, } \\
& Y(0)>0, K(0)>0, Y, K \in C^{1}\left(\left[0 ;+\infty\left[; \mathbb{R}_{+}\right),\right.\right.
\end{aligned}
$$

with $\left(a_{0}, C_{0}, C_{1}, C_{2}, m_{1}, m_{2}, \alpha\right) \in\left(\mathbb{R}_{+}^{*}\right)^{7}$ and $\left(a_{1}, a_{2}, b_{1}, b_{2}, \varrho\right) \in$ $\left(\mathbb{R}_{+}\right)^{5} . Y$ indicates the product and $K$ the stock of capital, and $\dot{Y}$ and $\dot{K}$ indicate, respectively, the increasing speed of the product and the stock of capital.

System (6) defined in this way is a more realistic system. It takes into account a great deal of economic observations of interactions between the product (GDP) and the stock of capital of an economy; namely,

(1) in the absence of the stock of capital, there will not be any explosion of the GDP because the increase of GDP becomes logistic so that, despite the technical progress, the economic production remains limited,

(2) in the absence of the production of the GDP, the economy will not be in short of capital if $b_{2}-\varrho>0$ because the evolution is as well logistic due to the diversification of the economy or the opportunities to convert a stock of physical capital in the stock of monetary capital,

(3) when the production (GDP) becomes abundant, there is a "saturation" of the ratio saving-capital and of the investment expressing the adoption of a nonwasting policy of the economic resources,

(4) when the production of the GDP is insufficient, the ratio saving-capital gets adapted and becomes proportional to the available GDP in order to avoid a shortage of production.

2.2. Presentation of Model (6) Reduced. In order to facilitate the qualitative study of system (6) that possesses 12 parameters $\left(a_{0}, a_{1}, a_{2}, b_{1}, b_{2}, C_{0}, C_{1}, C_{2}, m_{1}, m_{2}, \alpha, \varrho\right) \in\left(\mathbb{R}_{+}^{*}\right)^{12}$, let us change variables by reducing the number of parameters to 8.

\section{Definition of New Variables}

$$
\begin{gathered}
\tau=a_{0} t, \\
u(\tau)=\frac{Y(t)}{C_{0}}, \\
v(\tau)=\frac{m_{2}}{C_{0}} K(t) .
\end{gathered}
$$

\section{Definition of the Parameters}

$$
\begin{aligned}
& \beta_{1}=\frac{\alpha a_{1} C_{0}}{a_{0} m_{2}}, \\
& \beta_{2}=\frac{a_{2} C_{0}}{a_{0}}, \\
& \alpha_{1}=\frac{\alpha b_{1}}{a_{0}}, \\
& \alpha_{2}=\frac{b_{2}}{a_{0}}, \\
& d_{1}=\frac{C_{1}}{C_{0}}, \\
& d_{2}=\frac{C_{2}}{C_{0}} ; \\
& \gamma=\alpha \frac{m_{1}}{a_{0} m_{2}}, \\
& \delta=\frac{\varrho}{a_{0}} .
\end{aligned}
$$

System (6) becomes then the reduced system:

$$
\begin{aligned}
& \dot{u}(\tau)= {[1-u(\tau)] u(\tau) } \\
&+\left(\beta_{1} v(\tau)+\alpha_{1}\right)\left(1-\frac{v(\tau)}{u(\tau)+d_{2}}\right) u(\tau) \\
&-\frac{\gamma u(\tau) v(\tau)}{u(\tau)+d_{1}}, \\
& \dot{v}(\tau)=\left(\beta_{2} u(\tau)+\alpha_{2}\right)\left(1-\frac{v(\tau)}{u(\tau)+d_{2}}\right) v(\tau)-\delta v, \\
&(u, v) \in C^{1}\left(\mathbb{R}_{+}, \mathbb{R}^{2}\right) u(0)>0, v(0)>0,
\end{aligned}
$$

with $\left(d_{1}, d_{2}, \gamma\right) \in\left(\mathbb{R}_{+}^{*}\right)^{3}$ and $\left(\beta_{1}, \beta_{2}, \alpha_{1}, \alpha_{2}, \delta\right) \in\left(\mathbb{R}_{+}\right)^{5}$ so that $\left(\beta_{2}, \alpha_{2}\right) \neq(0,0)$.

\section{Boundness of Model (9) and Existence of a Positively Invariant Attracting Set}

In this section, we give the conditions of the boundness of the capital and the stock of capital justifying the fact that the economic resources are limited.

Lemma 3. The interior $\operatorname{int}\left(\mathbb{R}_{+}^{2}\right)$ and the boundary $\partial\left(\mathbb{R}_{+}^{2}\right)$ of the positive quadrant are, respectively, unvarying for system (9). 
Proof. Given $(u(0), v(0)) \in \mathbb{R}_{+}^{2}$, if $0 \leq \tau<+\infty$, due to the continuity of $u$ and $v$ over the compact $[0 ; \tau]$ then we have

$$
\begin{aligned}
& u(\tau)=u(0) \exp \left\{\int_{0}^{\tau}\left[1-u(t)+\left(\beta_{1} v(t)+\alpha_{1}\right)\left(1-\frac{v(t)}{u(t)+d_{2}}\right)-\frac{\gamma v(t)}{u(t)+d_{1}}\right] d t\right\}, \\
& v(\tau)=v(0) \exp \left\{\int_{0}^{\tau}\left[\left(\beta_{2} u(t)+\alpha_{2}\right)\left(1-\frac{v(t)}{u(t)+d_{2}}\right)-\delta\right] d t\right\} .
\end{aligned}
$$

So if $(u(0), v(0))=(0,0)$ then $(u(\tau), v(\tau))=(0 ; 0)$.

If $u(0)>0$ then $u(\tau)>0$ and if $v(0)>0$ then $v(\tau)>0$, $\forall \tau \in \mathbb{R}_{+}$.

Therefore $(u(0), v(0)) \in \partial\left(\mathbb{R}_{+}^{2}\right) \Rightarrow(u(\tau), v(\tau)) \in \partial\left(\mathbb{R}_{+}^{2}\right)$, $\forall \tau \in \mathbb{R}_{+}$, and $(u(0), v(0)) \in \operatorname{int}\left(\mathbb{R}_{+}^{2}\right) \Rightarrow(u(\tau), v(\tau)) \in$ $\operatorname{int}\left(\mathbb{R}_{+}^{2}\right), \forall \tau \in \mathbb{R}_{+}$.

Lemma 4 (cf. [6]). Given $(A, B) \in \mathbb{R}_{+}^{2}$ and $\phi$ continuous and derivable function so that there is $t_{0} \geq 0$ verifying $\phi\left(t_{0}\right)>0$, then, $\forall t \geq t_{0}$,

$$
\begin{aligned}
\frac{d \phi}{d t} & \leq B-A \phi \Longrightarrow \\
\phi(t) & \leq \frac{B}{A}-\left[\frac{B}{A}-\phi\left(t_{0}\right)\right] e^{-A\left(t-t_{0}\right)}, \\
\frac{d \phi}{d t} & \leq B-A \phi \Longrightarrow \\
\phi(t) & \leq \frac{B}{A}\left[1+\left(\frac{A \phi\left(t_{0}\right)}{B}-1\right) e^{-A\left(t-t_{0}\right)}\right]^{-1}, \\
\frac{d \phi}{d t} & \leq \phi(B-A \phi) \Longrightarrow \\
\phi(t) & \leq \frac{B}{A}\left[1+\left(\frac{B}{A \phi\left(t_{0}\right)}-1\right) e^{-B\left(t-t_{0}\right)}\right]^{-1}, \\
\frac{d \phi}{d t} & \geq \phi(B-A \phi) \Longrightarrow \\
\phi(t) & \geq \frac{B}{A}\left[1+\left(\frac{B}{A \phi\left(t_{0}\right)}-1\right) e^{-B\left(t-t_{0}\right)}\right]^{-1} .
\end{aligned}
$$

Definition 5 (see $[7,8])$. A solution $(u, v)=\phi\left(t, t_{0}, u_{0}, v_{0}\right)$ of (9) is said to be a boundary in $\mathbb{R}_{+}^{2}$, if there is compact $\mathscr{A}$ of $\mathbb{R}_{+}^{2}$ and a time $T\left(T=T\left(t_{0}, u_{0}, v_{0}\right)\right)$ so that $\forall\left(t_{0}, u_{0}, v_{0}\right) \in \mathbb{R} \times \mathbb{R}_{+}^{2}$, we have $(u, v)=\phi\left(t, t_{0}, u_{0}, v_{0}\right) \in \mathscr{A}$ for every $t \geq t_{0}$.

Theorem 6. Let us suppose that $0<\beta_{1}<4$. Let us set down

$$
\begin{aligned}
M_{u}= & \frac{4 d_{2} \beta_{1}+\left(\beta_{1} d_{2}+2 \alpha_{1}\right) \beta_{1} d_{2}+\alpha_{1}^{2}}{\beta_{1} d_{2}\left(4-\beta_{1}\right)}, \\
L_{1}= & \frac{\left(4+\left(4-\beta_{1}\right) M_{u}\right)^{2}}{16\left(4-\beta_{1}\right)} \\
& +\frac{\left(M_{u}+d_{2}\right)\left(\beta_{2} M_{u}+1+\alpha_{2}-\delta\right)^{2}}{4 \alpha_{2}} .
\end{aligned}
$$

Let us consider the following set:

$$
\mathscr{A}=\left\{(u, v) \in \mathbb{R}_{+}^{2}, 0 \leq u \leq M_{u}, 0 \leq u+v \leq L_{1}\right\} .
$$

(1) $\limsup [u(t)] \leq M_{u}$.

(2) $\limsup [u(t)+v(t)] \leq L_{1}$.

(3) $\mathscr{A}$ is unvarying for model (9).

(4) $\mathscr{A}$ is an attractive region for any solution of model (9) from the positive quadrant $\mathbb{R}_{+}^{2}$.

Proof. Let us consider system (9). Let us set down

$$
\begin{aligned}
f_{1}(u, v)= & {[1-u(\tau)] u(\tau) } \\
& +\left(\beta_{1} v(\tau)+\alpha_{1}\right)\left(1-\frac{v(\tau)}{u(\tau)+d_{2}}\right) u(\tau) \\
& -\frac{\gamma u(\tau) v(\tau)}{u(\tau)+d_{1}}, \\
f_{2}(u, v)= & \left(\beta_{2} u(\tau)+\alpha_{2}\right)\left(1-\frac{v(\tau)}{u(\tau)+d_{2}}\right) v(\tau)-\delta v .
\end{aligned}
$$

(1) Let us show that $\limsup [u(t)] \leq M_{u}=\left(4 d_{2} \beta_{1}+\right.$ $\left.\left(\beta_{1} d_{2}+2 \alpha_{1}\right) \beta_{1} d_{2}+\alpha_{1}^{2}\right) / \beta_{1} d_{2}\left(4-\beta_{1}\right)$. We have $d u / d t=$ $(1-u) u+\left(\beta_{1} v+\alpha_{1}\right)\left(1-v /\left(u+d_{2}\right)\right) u-\gamma u v /\left(u+d_{1}\right)$ and $\max _{v}\left\{\left(\beta_{1} v+\alpha_{1}\right)\left(1-v /\left(u+d_{2}\right)\right) u\right\}=u\left[\beta_{1}\left(u+d_{2}\right)+\right.$ $\left.\alpha_{1}\right]^{2} / 4 \beta_{1}\left(u+d_{2}\right)$. Then, $d u / d t \leq\left(B_{1}-A_{1} u\right) u$ with $B_{1}=\left(4 d_{2} \beta_{1}+\left(\beta_{1} d_{2}+2 \alpha_{1}\right) \beta_{1} d_{2}+\alpha_{1}^{2}\right) / 4 \beta_{1} d_{2}$ and $A_{1}=$ $\left(4-\beta_{1}\right) / 4$. Then through the application property (13) of Lemma 4, we have $\forall t \geq 0, u(t) \leq M_{u}[1+$ $\left.\left(B_{1} / A_{1} u(0)-1\right) e^{-B_{1} t}\right]^{-1}$ with $M_{u}=B_{1} / A_{1}$. Therefore, $\forall \varepsilon>0, \exists T_{1}>0$ so that $\forall t>T_{1}, u(t) \leq M_{u}+\varepsilon$.

Then $\lim \sup [u(t)] \leq M_{u}$

$$
=\frac{4 d_{2} \beta_{1}+\left(\beta_{1} d_{2}+2 \alpha_{1}\right) \beta_{1} d_{2}+\alpha_{1}^{2}}{\beta_{1} d_{2}\left(4-\beta_{1}\right)} .
$$

So $\forall t>T_{1}, u(t) \leq M_{u}$.

(2) Let us prove that $\limsup [u(t)+v(t)] \leq L_{1}$. Let us set down $S(t)=u(t)+v(t)$. We have $d u / d t \leq\left(B_{1}-A_{1} u\right) u$ 
and $d v / d t=\left[\beta_{2} u+\alpha_{2}-\delta-\left(\beta_{2} u /\left(u+d_{2}\right)\right) v-\left(\alpha_{2} /(u+\right.\right.$ $\left.\left.\left.d_{2}\right)\right) v\right] v \leq\left[\left(\beta_{2} M_{u}+\alpha_{2}-\delta\right)-\left(\alpha_{2} /\left(M_{u}+d_{2}\right)\right) v\right] v$; then, we have $d S / d t+S(t) \leq\left(1+B_{1}-A_{1} u\right) u+[(1+$ $\left.\left.\beta_{2} M_{u}+\alpha_{2}-\delta\right)-\left(\alpha_{2} /\left(M_{u}+d_{2}\right)\right) v\right] v$. Now $\forall a, b \in \mathbb{R}_{+}^{*}$, $\max _{x \geq 0}(b-a x) x=b^{2} / 4 a$. Then $d S / d t+S(t) \leq L_{1}=$ $\left(4+\left(4-\beta_{1}\right) M_{u}\right)^{2} / 16\left(4-\beta_{1}\right)+\left(M_{u}+d_{2}\right)\left(\beta_{2} M_{u}+1+\alpha_{2}-\right.$ $\delta)^{2} / 4 \alpha_{2}$. Let us set down $A_{2}=1>0$ and $B_{2}=L_{1}>0$ then, $d S / d t \leq\left(B_{2}-A_{2} S\right)$. Now $S(0)>0$; then, through the application property (12) of Lemma 4, we have $\forall t \geq 0, S(t) \leq L_{1}\left[1+\left(S(0) / L_{1}-1\right) e^{-t}\right]^{-1}$; then $\forall \varepsilon>0, \exists T_{2}>0$ so that $\forall t>T_{2}, S(t) \leq L_{1}+\varepsilon$.

So limsup $[S(t)] \leq L_{1}$.

$$
\text { Consequently } \forall t \geq T_{2}, u(t)+v(t) \leq L_{1} \text {. }
$$

(3) Let us show that $\mathscr{A}$ is unvarying for model (9). Given $(u(0), v(0)) \in \mathscr{A}$, from Lemma 3 , of (18) and (19), $\forall t \geq T=\max \left(T_{1}, T_{2}\right),(u(0), v(0)) \in \operatorname{int}(\mathscr{A}) \Rightarrow$ $\left.(u(t), v(t)) \in \mathscr{A},(u(0), v(0)) \in\{0\} \times] 0 ; L_{1}\right] \Rightarrow$ $\left.(u(t), v(t)) \in\{0\} \times] 0 ; L_{1}\right] \subset \mathscr{A}$, and $(u(0), v(0)) \in$ ] $\left.\left.\left.0 ; M_{u}\right] \times\{0\} \Rightarrow(u(t), v(t)) \in\right] 0 ; M_{u}\right] \times\{0\} \subset \mathscr{A}$. So $\mathscr{A}$ is unvarying for model (9).

(4) Let us show that $\mathscr{A}$ is an attractive solution of model (9). Let us show that $(u(0), v(0)) \in \mathbb{R}_{+}^{2}$. We deduce from Lemma 3, (18), and (19) that $\forall t \geq T=$ $\max \left(T_{1}, T_{2}\right),(u(0), v(0)) \in \operatorname{int}\left(\mathbb{R}_{+}^{2}\right) \Rightarrow(u(t), v(t)) \in$ $\mathscr{A}$, and $(u(0), v(0)) \in \partial\left(\mathbb{R}_{+}^{2}\right) \Rightarrow(u(t), v(t)) \in \mathscr{A}$, $\forall t \geq T$. Consequently $\mathscr{A}$ is an attractive region for any solution of model (9) from the positive quadrant $\mathbb{R}_{+}^{2}$.

\section{Equilibrium of Model (9)}

We are now going to give the conditions of a balanced growth (stationary) of the product and the stock of capital and the quantitative values of the parameters in equilibrium.

\subsection{Case in Which $\left(\beta_{1}, \beta_{2}\right) \neq(0,0)$}

\subsubsection{Trivial Equilibrium}

Proposition 7. (1) If $0<\alpha_{2} \leq \delta$ then system (9) admits two trivial equilibriums:

$$
\begin{aligned}
& U_{0}^{*}=(0 ; 0), \\
& U_{1}^{*}=\left(1+\alpha_{1} ; 0\right) .
\end{aligned}
$$

(2) If $\alpha_{2}>\delta>0$ then system (9) admits three trivial equilibriums:

$$
\begin{aligned}
& U_{0}^{*}=(0 ; 0), \\
& U_{1}^{*}=\left(1+\alpha_{1} ; 0\right), \\
& U_{2}^{*}=\left(0 ; \frac{\left(\alpha_{2}-\delta\right) d_{2}}{\alpha_{2}}\right) .
\end{aligned}
$$

\subsubsection{Interior Equilibrium}

Theorem 8. Let $P(x)=a_{4} x^{4}+a_{3} x^{3}+a_{2} x^{2}+a_{1} x+a_{0}$ with

$$
\begin{aligned}
a_{4}= & -\beta_{2}^{2}, \\
a_{3}= & \left(1-d_{1}\right) \beta_{2}^{2}-2 \beta_{2} \alpha_{2}+\beta_{2}\left(\beta_{1} \delta-\gamma \beta_{2}\right), \\
a_{2}= & 2 \beta_{2} \alpha_{2}\left(1-d_{1}\right)-\alpha_{2}^{2}+d_{1} \beta_{2}^{2}+\alpha_{1} \delta \beta_{2}-\gamma \beta_{2} \alpha_{2} \\
& +\beta_{1} \beta_{2} \delta d_{1}+\left(\beta_{2} d_{2}+\alpha_{2}-\delta\right)\left(\beta_{1} \delta-\gamma \beta_{2}\right), \\
a_{1}= & \alpha_{1} \delta\left(\beta_{2} d_{1}+\alpha_{2}\right)+\left(1-d_{1}\right) \alpha_{2}^{2}+2 \beta_{2} \alpha_{2} d_{1} \\
& +\left(\beta_{1} \delta-\gamma \beta_{2}\right) d_{2}\left(\alpha_{2}-\delta\right) \\
& +\left(\beta_{1} \delta d_{1}-\gamma \alpha_{2}\right)\left(\beta_{2} d_{2}+\alpha_{2}-\delta\right), \\
a_{0}= & d_{1} \alpha_{2}^{2}+\alpha_{1} \alpha_{2} \delta d_{1}+\left(\beta_{1} \delta d_{1}-\gamma \alpha_{2}\right) d_{2}\left(\alpha_{2}-\delta\right) .
\end{aligned}
$$

(1) System (9) does not admit the interior equilibrium if $\beta_{2} M_{u}+\alpha_{2}-\delta<0$.

(2) Any interior equilibrium $E^{*}=\left(u^{*} ; v^{*}\right)$ of system (9) satisfies the following relations:

$$
\begin{aligned}
P\left(u^{*}\right) & =0, \\
v^{*} & =\frac{\left(\beta_{2} u^{*}+\alpha_{2}-\delta\right)}{\beta_{2} u^{*}+\alpha_{2}}\left(u^{*}+d_{2}\right)
\end{aligned}
$$

$$
\text { with } \beta_{2} u^{*}+\alpha_{2}-\delta>0 \text {. }
$$

Proof. Let us consider model (9); then, $\left(\beta_{2}, \alpha_{2}\right) \neq(0,0)$. Given $E^{*}=\left(u^{*} ; v^{*}\right)$ an equilibrium of the model (9),

(1) if $\beta_{2} M_{u^{*}}+\alpha_{2}-\delta<0$ then $\beta_{2} u^{*}+\alpha_{2}-\delta<0$ so for any $u^{*} \geq 0$, we have $v^{*}<0$; therefore the system does not admit any interior equilibrium.

(2) Given $E^{*}=\left(u^{*} ; v^{*}\right) \in\left(\mathbb{R}_{+}^{*}\right)^{2}$ an interior equilibrium, then we have

$$
\begin{aligned}
& \left(1-u^{*}\right)+\left(\beta_{1} v^{*}+\alpha_{1}\right)\left(1-\frac{v^{*}}{u^{*}+d_{2}}\right)-\frac{\gamma v^{*}}{u^{*}+d_{1}} \\
& \quad=0
\end{aligned}
$$


Therefore,

$$
\begin{aligned}
& \frac{\left(1-u^{*}\right)\left(\beta_{2} u^{*}+\alpha_{2}\right)^{2}\left(u^{*}+d_{1}\right)+\alpha_{1} \delta\left(\beta_{2} u^{*}+\alpha_{2}\right)\left(u^{*}+d_{1}\right)+\left[\beta_{1} \delta\left(u^{*}+d_{1}\right)-\gamma\left(\beta_{2} u^{*}+\alpha_{2}\right)\right]\left(\beta_{2} u^{*}+\alpha_{2}-\delta\right)\left(u^{*}+d_{2}\right)}{\left(\beta_{2} u^{*}+\alpha_{2}\right)^{2}\left(u^{*}+d_{1}\right)} \\
& \quad=0 \\
& v^{*}=\frac{\left(\beta_{2} u^{*}+\alpha_{2}-\delta\right)}{\beta_{2} u^{*}+\alpha_{2}}\left(u^{*}+d_{2}\right) .
\end{aligned}
$$

Posing

$$
\begin{aligned}
a_{4}= & -\beta_{2}^{2}, \\
a_{3}= & \left(1-d_{1}\right) \beta_{2}^{2}-2 \beta_{2} \alpha_{2}+\beta_{2}\left(\beta_{1} \delta-\gamma \beta_{2}\right) \\
a_{2}= & 2 \beta_{2} \alpha_{2}\left(1-d_{1}\right)-\alpha_{2}^{2}+d_{1} \beta_{2}^{2}+\alpha_{1} \delta \beta_{2}-\gamma \beta_{2} \alpha_{2} \\
& +\beta_{1} \beta_{2} \delta d_{1}+\left(\beta_{2} d_{2}+\alpha_{2}-\delta\right)\left(\beta_{1} \delta-\gamma \beta_{2}\right),
\end{aligned}
$$

$$
\begin{aligned}
a_{1}= & \alpha_{1} \delta\left(\beta_{2} d_{1}+\alpha_{2}\right)+\left(1-d_{1}\right) \alpha_{2}^{2}+2 \beta_{2} \alpha_{2} d_{1} \\
& +\left(\beta_{1} \delta-\gamma \beta_{2}\right) d_{2}\left(\alpha_{2}-\delta\right) \\
& +\left(\beta_{1} \delta d_{1}-\gamma \alpha_{2}\right)\left(\beta_{2} d_{2}+\alpha_{2}-\delta\right), \\
a_{0}= & d_{1} \alpha_{2}^{2}+\alpha_{1} \alpha_{2} \delta d_{1}+\left(\beta_{1} \delta d_{1}-\gamma \alpha_{2}\right) d_{2}\left(\alpha_{2}-\delta\right)
\end{aligned}
$$

and $P(x)=a_{4} x^{4}+a_{3} x^{3}+a_{2} x^{2}+a_{1} x+a_{0}$, we obtain

$$
E^{*}=\left(u^{*} ; v^{*}\right) \in\left(\mathbb{R}_{+}^{*}\right)^{2} \Longleftrightarrow\left\{\begin{array}{l}
P\left(u^{*}\right)=0, \\
v^{*}=\frac{\left(\beta_{2} u^{*}+\alpha_{2}-\delta\right)}{\beta_{2} u^{*}+\alpha_{2}}\left(u^{*}+d_{2}\right) \quad \text { with } \beta_{2} u^{*}+\alpha_{2}-\delta>0 .
\end{array}\right.
$$

Corollary 9. Let us suppose that $\alpha_{2}-\delta>0$ and considering the polynomial $P(x)$ defined in Theorem 8 , let us set down $p=$ $a_{4} a_{2}-3 a_{3}^{2} / 8, q=a_{3}^{3} / 8-a_{4} a_{3} a_{2} / 2+a_{4}^{2} a_{1}, r=a_{3}^{3} / 8-a_{4} a_{3} a_{2} / 4+$ $a_{4} a_{3}^{2} a_{2} / 16-3 a_{3}^{3} / 4^{4}, A=4 r+p^{2} / 3, B=-q^{2}-2 p^{3} / 27+8 p r / 3$, and $\Delta=27 B^{2}-4 A^{3}$.

System (9) admits a unique interior equilibrium $E^{*}=$ $\left(u^{*}, v^{*}\right)$ such that $P\left(u^{*}\right)=0$ and $v^{*}=\left(\left(\beta_{2} u^{*}+\alpha_{2}-\delta\right) /\left(\beta_{2} u^{*}+\right.\right.$ $\left.\left.\alpha_{2}\right)\right)\left(u^{*}+d_{2}\right)$ in each of the following cases:

(a) $\Delta>0, a_{0}>0$.

(b) $\Delta=0$ and one of the following conditions is verified:

(i) $a_{0}>0, p<0, q>0$ and $a_{3}<0$.

(ii) $a_{0}<0, p>0, q>0$ and $a_{3}>0$.

(iii) $a_{0}<0, p>0, q<0, a_{3}<0$, and $a_{3}^{2} / 4<$ $-(2 \sqrt[3]{B / 2}+2 p / 3)$

(iv) $a_{0}>0, p<0, q<0, a_{3}<0$ and $-(2 \sqrt[3]{B / 2}+$ $2 p / 3)<a_{3}^{2} / 4$.

(v) $a_{0}<0, p>0, q<0, a_{3}>0$ and $-(2 \sqrt[3]{B / 2}+$ $2 p / 3)<a_{3}^{2} / 4$.
Proof. Given $P(x)=a_{4} x^{4}+a_{3} x^{3}+a_{2} x^{2}+a_{1} x+a_{0}$ the polynomial is defined in Theorem 8 and $E^{*}=\left(u^{*}, v^{*}\right) \in\left(\mathbb{R}_{+}^{*}\right)^{2}$ such that $P\left(u^{*}\right)=0$ and $v^{*}=\left(\left(\beta_{2} u^{*}+\alpha_{2}-\delta\right) /\left(\beta_{2} u^{*}+\alpha_{2}\right)\right)\left(u^{*}+\right.$ $d_{2}$ ).

Let us pose $x=\left(1 / a_{4}\right)\left(y-a_{3} / 4\right)$; then, the equation $P(x)=0$ will be reduced to $\left(E_{1}\right): y^{4}+p y^{2}+q y+r=$ 0 with $p=a_{4} a_{2}-3 a_{3}^{2} / 8, q=a_{3}^{3} / 8-a_{4} a_{3} a_{2} / 2+a_{4}^{2} a_{1}$, $r=a_{3}^{3} / 8-a_{4} a_{3} a_{2} / 4+a_{4} a_{3}^{2} a_{2} / 16-3 a_{3}^{3} / 4^{4}$. The characteristic equation of $\left(E_{1}\right)$ is $\left(E_{2}\right): u^{3}-2 p u^{2}+\left(p^{2}-4 r\right) u+q^{2}=0$. Let us pose $A=4 r+p^{2} / 3, B=-q^{2}-2 p^{3} / 27+8 p r / 3$, $\Delta=27 B^{2}-4 A^{3}$. Given, $u_{1}, u_{2}, u_{3}$ solutions of $\left(E_{2}\right)$ certifying $\sqrt{-u_{1}} \times \sqrt{-u_{2}} \times \sqrt{-u_{3}}=-q$, then, $y_{1}, y_{2}, y_{3}$, and $y_{4}$, the solutions in $\mathbb{C}$ of $\left(E_{1}\right)$, are $y_{1}=(1 / 2)\left(\sqrt{-u_{1}}+\sqrt{-u_{2}}+\sqrt{-u_{3}}\right)$, $y_{2}=(1 / 2)\left(\sqrt{-u_{1}}-\sqrt{-u_{2}}-\sqrt{-u_{3}}\right), y_{3}=(1 / 2)\left(-\sqrt{-u_{1}}+\sqrt{-u_{2}}-\right.$ $\left.\sqrt{-u_{3}}\right), y_{4}=(1 / 2)\left(-\sqrt{-u_{1}}-\sqrt{-u_{2}}+\sqrt{-u_{3}}\right)$.

So, the roots in $\mathbb{C}$ of $P(x)$ are $x_{i}=\left(1 / a_{4}\right)\left(y_{i}-a_{3} / 4\right)$ for $i \in\{1,2,3,4\}$.

The roots $x_{1}, x_{2}, x_{3}$, and $x_{4}$ in $\mathbb{C}$ of $P(x)$ prove the following system: $x_{1} x_{2} x_{3} x_{4}=a_{0} / a_{4}$ and $x_{1}+x_{2}+x_{3}+x_{4}=$ $-a_{3} / a_{4}$.

By examining the number of positive roots of $P(x)$ and knowing that $P\left(u^{*}\right)=0$ and $v^{*}=\left(\left(\beta_{2} u^{*}+\alpha_{2}-\delta\right) /\left(\beta_{2} u^{*}+\right.\right.$ $\left.\left.\alpha_{2}\right)\right)\left(u^{*}+d_{2}\right)>0$ because $\alpha_{2}-\delta>0$, we get Corollary 9 . 
4.2. Case in Which $\left(\beta_{1}, \beta_{2}\right)=(0,0)$. Considering the conditions $\left(\beta_{1}, \beta_{2}\right)=(0,0)$ and $\alpha_{1} \neq 0, \alpha_{2} \neq 0$ in system (9), we get the following system:

$$
\begin{gathered}
\dot{u}(\tau)=[1-u(\tau)] u(\tau)+\alpha_{1}\left(1-\frac{v(\tau)}{u(\tau)+d_{2}}\right) u(\tau) \\
-\frac{\gamma u(\tau) v(\tau)}{u(\tau)+d_{1}}, \\
\dot{v}(\tau)=\alpha_{2}\left(1-\frac{v(\tau)}{u(\tau)+d_{2}}\right) v(\tau)-\delta v, \\
(u, v) \in C^{1}\left(\mathbb{R}_{+}, \mathbb{R}^{2}\right), u(0)>0, v(0)>0,
\end{gathered}
$$

with $\left(d_{1}, d_{2}, \alpha_{1}, \alpha_{2}, \gamma\right) \in\left(\mathbb{R}_{+}^{*}\right)^{5}$ and $\delta \in \mathbb{R}_{+}$.

\subsubsection{Trivial Equilibrium}

Proposition 10. (1) If $\alpha_{2} \leq \delta$, then, system (28) admits two trivial equilibriums:

$$
\begin{aligned}
& U_{0}^{*}=(0 ; 0), \\
& U_{1}^{*}=\left(1+\alpha_{1} ; 0\right) .
\end{aligned}
$$

(2) If $\alpha_{2}>\delta$, then, system (28) admits three trivial equilibriums:

$$
\begin{aligned}
& U_{0}^{*}=(0 ; 0), \\
& U_{1}^{*}=\left(1+\alpha_{1} ; 0\right), \\
& U_{2}^{*}=\left(0 ; \frac{\left(\alpha_{2}-\delta\right) d_{2}}{\alpha_{2}}\right) .
\end{aligned}
$$

\subsubsection{Interior Equilibrium}

Theorem 11. Given that $P(x)=a_{2} x^{2}+a_{1} x+a_{0}$ with

$$
\begin{aligned}
& a_{2}=-\alpha_{2}^{2}, \\
& a_{1}=\alpha_{2}^{2}\left(1-d_{1}\right)+\delta \alpha_{1} \alpha_{2}-\gamma \alpha_{2}\left(\alpha_{2}-\delta\right), \\
& a_{0}=\alpha_{2}^{2} d_{1}+\delta \alpha_{1} \alpha_{2} d_{1}-\gamma \alpha_{2} d_{2}\left(\alpha_{2}-\delta\right)
\end{aligned}
$$

(1) if $\alpha_{2}-\delta \leq 0$ then system (28) does not admit any interior equilibriums,

(2) if $\alpha_{2}-\delta>0$ then any interior equilibrium $E^{*}=\left(u^{*} ; v^{*}\right)$ of system (28) verifies the following system:

$$
\begin{aligned}
P\left(u^{*}\right) & =0, \\
v^{*} & =\frac{\left(\alpha_{2}-\delta\right)}{\alpha_{2}}\left(u^{*}+d_{2}\right) .
\end{aligned}
$$

Corollary 12. Let us suppose that $\alpha_{2}-\delta>0$ and consider the polynomial $P(x)$ of Theorem 11. System (28) admits a unique interior equilibrium $E^{*}=\left(u^{*}, v^{*}\right)$ so that $u^{*}=\left(a_{1}+\right.$ $\left.\sqrt{a_{1}^{2}+4 \alpha_{2}^{2} a_{0}}\right) / 2 \alpha_{2}^{2}$ and $v^{*}=\left(\left(\alpha_{2}-\delta\right) / \alpha_{2}\right)\left(u^{*}+d_{2}\right)$ if one of the following conditions is verified:

(i) $\delta>\alpha_{2}\left(\gamma d_{2}-d_{1}\right) /\left(\alpha_{1} d_{1}+\gamma d_{2}\right)$. (ii) $\delta<\alpha_{2}\left(\gamma d_{2}-d_{1}\right) /\left(\alpha_{1} d_{1}+\gamma d_{2}\right), a_{1}^{2}+4 \alpha_{2}^{2} a_{0}=0$, and $a_{1}>0$.

(iii) $\delta=\alpha_{2}\left(\gamma d_{2}-d_{1}\right) /\left(\alpha_{1} d_{1}+\gamma d_{2}\right)$ and $a_{1}>0$.

Proof. If $E^{*}=\left(u^{*}, v^{*}\right)$ is an interior equilibrium of (28) and $P(x)=-\alpha_{2}^{2} x^{2}+a_{1} x+a_{0}$, the polynomial, $a_{0}$ and $a_{1}$ are stipulated in Theorem 11. Then, $P\left(u^{*}\right)=0$ and $v^{*}=$ $\left(\left(\alpha_{2}-\delta\right) / \alpha_{2}\right)\left(u^{*}+d_{2}\right)$.

We get $a_{0}=\alpha_{2}^{2} d_{1}+\delta \alpha_{1} \alpha_{2} d_{1}-\gamma \alpha_{2} d_{2}\left(\alpha_{2}-\delta\right)=0 \Leftrightarrow \delta=$ $\alpha_{2}\left(\gamma d_{2}-d_{1}\right) /\left(\alpha_{1} d_{1}+\gamma d_{2}\right)$.

By examining the number of positive roots of $P(x)$ in which $a_{0}=0$ and $a_{0} \neq 0$, we obtain researched results.

\section{Local Stability and Permanence of Model (9)}

In this section, we first define the conditions in which this balanced growth of the product and the stock of capital of the economy are stable or unstable. Then, let us examine the possibility of having permanently those two parameters of the economy (sustainable development). This permanence of the product and the stock of capital of the economy are noticed either through the convergence (of both parameters) towards a stable equilibrium or through a fluctuation of both parameters around an unstable equilibrium, that is, the convergence towards a limited cycle.

5.1. Local Stability of Model (9). In system (9), we pose the following:

$$
\begin{aligned}
f_{1}(u, v)= & {[1-u(\tau)] u(\tau) } \\
& +\left(\beta_{1} v(\tau)+\alpha_{1}\right)\left(1-\frac{v(\tau)}{u(\tau)+d_{2}}\right) u(\tau) \\
& -\frac{\gamma u(\tau) v(\tau)}{u(\tau)+d_{1}}, \\
f_{2}(u, v)= & \left(\beta_{2} u(\tau)+\alpha_{2}\right)\left(1-\frac{v(\tau)}{u(\tau)+d_{2}}\right) v(\tau) \\
& -\delta v .
\end{aligned}
$$

We have $\dot{u}(\tau)=f_{1}(u, v)$ and $\dot{v}(\tau)=f_{2}(u, v)$.

Let us note that $J(E)$, the Jacobian matrix of the system, is linear around $E=(u, v)$. Then, we have

$$
J(E)=\left(\begin{array}{ll}
\frac{\partial f_{1}}{\partial u}(E) & \frac{\partial f_{1}}{\partial v}(E) \\
\frac{\partial f_{2}}{\partial u}(E) & \frac{\partial f_{2}}{\partial v}(E)
\end{array}\right)=\left(\begin{array}{lll}
J_{11}(E) & J_{12}(E) \\
J_{21}(E) & J_{22}(E)
\end{array}\right)
$$

with

$$
\begin{gathered}
\frac{\partial f_{1}}{\partial u}=1-2 u+\left(\beta_{1} v+\alpha_{1}\right)\left(1-\frac{v}{u+d_{2}}\right) \\
+\frac{\left(\beta_{1} v+\alpha_{1}\right) u v}{\left(u+d_{2}\right)^{2}}-\frac{\gamma d_{1} v}{\left(u+d_{1}\right)^{2}},
\end{gathered}
$$




$$
\begin{aligned}
& \frac{\partial f_{1}}{\partial v}=\left[\beta_{1}-\frac{2 \beta_{1}}{u+d_{2}} v-\frac{\alpha_{1}}{u+d_{2}}-\frac{\gamma}{u+d_{1}}\right] u, \\
& \frac{\partial f_{2}}{\partial u}=\left[\beta_{2}\left(1-\frac{v}{u+d_{2}}\right)+\frac{\left(\beta_{2} u+\alpha_{2}\right) v}{\left(u+d_{2}\right)^{2}}\right] v, \\
& \frac{\partial f_{2}}{\partial v}=\left(\beta_{2} u+\alpha_{2}\right)\left(1-\frac{2 v}{u+d_{2}}\right)-\delta .
\end{aligned}
$$

Theorem 13 (local stability). (1) Stability of $U_{0}^{*}=(0 ; 0)$ :

(a) $U_{0}^{*}$ is an unstable node if $\alpha_{2}>\delta$.

(b) $U_{0}^{*}$ is an unstable saddle point if $\alpha_{2}<\delta$,

(i) repulsive along the direction $u$,

(ii) attractive along the direction $v$.

(2) Stability of $U_{1}^{*}=\left(1+\alpha_{1} ; 0\right)$ :

(a) $U_{1}^{*}$ is stable if $\alpha_{2}+\left(1+\alpha_{1}\right) \beta_{2}<\delta$.

(b) $U_{1}^{*}$ is an unstable saddle point if $\alpha_{2}+\left(1+\alpha_{1}\right) \beta_{2}>\delta$,

(i) attractive along the direction $u$,

(ii) repulsive along the direction $w_{1}$.

(3) Stability of $U_{2}^{*}=\left(0 ;\left(\alpha_{2}-\delta\right) d_{2} / \alpha_{2}\right)$ for $\alpha_{2}>\delta$ :

(a) $U_{2}^{*}$ is stable if $Q(\delta)<0$.

(b) $U_{2}^{*}$ is an unstable saddle point if $Q(\delta)>0$, repulsive along the direction $w_{2}$ and attractive along the direction $v$.

(4) Given $E^{*}=\left(u^{*} ; v^{*}\right)$, an interior equilibrium of (9) verifying system (23) of Theorem 8 , and $J$ its associate Jacobian matrix,

(a) $E^{*}$ is stable (node or a focus) if $\operatorname{det}(J)>0$ and $\operatorname{Tr}(J)<$ 0 ,

(b) $E^{*}$ is marginal or a center if $\operatorname{det}(J) \geq 0$ and $\operatorname{Tr}(J)=0$,

(c) $E^{*}$ is unstable if $\operatorname{det}(J)<0$ or $\operatorname{det}(J)>0$ and $\operatorname{Tr}(J)>0$; more precisely,

(i) $E^{*}$ is a node or a focus if $\operatorname{det}(J)>0$ and $\operatorname{Tr}(J)>0$,

(ii) $E^{*}$ is a unstable saddle if $\operatorname{det}(J)<0$.

With $\operatorname{Tr}(J)$ the trace and $\operatorname{det}(J)$ the determinant of $J$, the polynomial $Q(x)=-d_{2} \beta_{1} d_{1} x^{2}+\left[\gamma \alpha_{2} d_{2}+d_{2} d_{1} \beta_{1} \alpha_{2}+\right.$ $\left.d_{1} \alpha_{1} \alpha_{2}\right] x+\alpha_{2}^{2}\left[d_{1}-\gamma d_{2}\right]$, the vectors $w_{1}=\left(\left(1+\alpha_{1}\right)\left[\beta_{1}-\alpha_{1} /(1+\right.\right.$ $\left.\left.\left.\alpha_{1}+d_{2}\right)-\gamma /\left(1+\alpha_{1}+d_{1}\right)\right] ;\left(1+\alpha_{1}\right)\left(\beta_{2}+1\right)+\alpha_{2}-\delta\right)$, and $w_{2}=\left(Q(\delta) / d_{1} \alpha_{2}^{2}+\alpha_{2}-\delta ;\left[\beta_{2} d_{2} \delta+\alpha_{2}\left(\alpha_{2}-\delta\right)\right]\left(\alpha_{2}-\delta\right) / \alpha_{2}^{2}\right)$.

Proof. Given $J_{k}$, the Jacobian matrix of the system, is linear around the equilibrium $U_{k}^{*}$ for $k \in\{0,1,2\}$ and $J$ of $E^{*}=$ $\left(u^{*}, v^{*}\right)$, we have the following:

(1) Stability of $U_{0}^{*}=(0 ; 0)$ :
We have

$$
\begin{aligned}
U_{0}^{*} & =(0 ; 0) \Longrightarrow \\
J_{0} & =\left(\begin{array}{cc}
1+\alpha_{1} & 0 \\
0 & \alpha_{2}-\delta
\end{array}\right) .
\end{aligned}
$$

The numbers $\lambda_{1}=1+\alpha_{1}>0$ and $\lambda_{2}=\alpha_{2}-\delta$ are the eigenvalues of $J_{0}$. Eigenspace associated with $\lambda_{1}$ is $E_{\lambda_{1}}^{(0)}=\langle(1 ; 0)\rangle$, where $\left\langle\left\{r_{k}\right\}_{1 \leq k \leq N}\right\rangle$ indicates the vectorial subspace generated by the family: $\left\{r_{k}\right\}_{1 \leq k \leq N^{*}}$ with $N \in \mathbb{N}$. The eigenspace associated with $\lambda_{2}$ is $E_{\lambda_{2}}^{(0)}=\langle(0 ; 1)\rangle$. We have the following:

(a) If $\alpha_{2}>\delta$ then $\lambda_{1}>0$ and $\lambda_{2}>0$; then $U_{0}^{*}$ is an unstable node and its unstable manifold is $E^{u}=\langle(1 ; 0),(0 ; 1)\rangle=\mathbb{R}^{2}$.

(b) If $\alpha_{2}<\delta$ then $\lambda_{1}>0$ and $\lambda_{2}<0$; then $U_{0}^{*}$ is an unstable saddle point, the unstable manifold of which is $E^{u}=\langle(1 ; 0)\rangle$ and the stable manifold is $E^{\mathcal{S}}=\langle(0 ; 1)\rangle$.

(c) If $\alpha_{2}=\delta$ then $\lambda_{1}>0$ and $\lambda_{2}=0$; therefore $U_{0}^{*}$ is an equilibrium the unstable manifold of which is $E^{\mathcal{u}}=\langle(1 ; 0)\rangle$ and of central manifold is $E^{\mathcal{C}}=\langle(0 ; 1)\rangle$.

(2) Stability of $U_{1}^{*}=\left(1+\alpha_{1} ; 0\right)$ :

We have

$J_{1}$

$$
=\left(\begin{array}{cc}
-\left(1+\alpha_{1}\right) & \left(1+\alpha_{1}\right)\left[\begin{array}{c}
\left.\beta_{1}-\frac{\alpha_{1}}{1+\alpha_{1}+d_{2}}-\frac{\gamma}{1+\alpha_{1}+d_{1}}\right] \\
0
\end{array}\right. \\
\left(1+\alpha_{1}\right) \beta_{2}+\alpha_{2}-\delta
\end{array}\right) .
$$

So $\lambda_{1}=-\left(1+\alpha_{1}\right)<0$ and $\lambda_{2}=\left(1+\alpha_{1}\right) \beta_{2}+\alpha_{2}-\delta$ are the eigenvalues of $J_{1}$.

The eigenspace associated with $\lambda_{1}$ is $E_{\lambda_{1}}^{(1)}=\langle(1 ; 0)\rangle$.

Note that $w_{1}=\left(\left(1+\alpha_{1}\right)\left[\beta_{1}-\alpha_{1} /\left(1+\alpha_{1}+d_{2}\right)-\gamma /(1+\right.\right.$ $\left.\left.\left.\alpha_{1}+d_{1}\right)\right] ;\left(1+\alpha_{1}\right)\left(\beta_{2}+1\right)+\alpha_{2}-\delta\right)$.

The eigenspace associated with $\lambda_{2}$ is $E_{\lambda_{2}}^{(1)}=\left\langle w_{1}\right\rangle$.

We get the following:

(a) If $\alpha_{2}+\left(1+\alpha_{1}\right) \beta_{2}<\delta$ then $\lambda_{1}<0$ and $\lambda_{2}<0$; then $U_{1}^{*}$ is stable and its stable manifold is $E^{u}=$ $\mathbb{R}^{2}$.

(b) If $\alpha_{2}+\left(1+\alpha_{1}\right) \beta_{2}>\delta$ then $\lambda_{1}<0$ and $\lambda_{2}>0$; then $U_{1}^{*}$ is an unstable saddle point of which the stable manifold is $E^{s}=\langle(1 ; 0)\rangle$ and the unstable manifold is $E^{\mathfrak{u}}=\left\langle w_{1}\right\rangle$.

(c) If $\alpha_{2}+\left(1+\alpha_{1}\right) \beta_{2}=\delta$ then $\lambda_{1}<0$ and $\lambda_{2}=0$; consequently $U_{1}^{*}$ is an equilibrium the stable manifold of which is $E^{\mathcal{S}}=\langle(1 ; 0)\rangle$ and the central manifold is $E^{c}=\left\langle w_{1}\right\rangle$.

(3) Stability of $U_{2}^{*}=\left(0 ;\left(\alpha_{2}-\delta\right) d_{2} / \alpha_{2}\right)$ : 
We have

$J_{2}$

$=\left(\begin{array}{cc}\frac{Q(\delta)}{d_{1} \alpha_{2}^{2}} & 0 \\ \frac{\left[\beta_{2} d_{2} \delta+\alpha_{2}\left(\alpha_{2}-\delta\right)\right]\left(\alpha_{2}-\delta\right)}{\alpha_{2}^{2}} & -\left(\alpha_{2}-\delta\right)\end{array}\right)$.

So $\lambda_{1}=Q(\delta) / d_{1} \alpha_{2}^{2}$ and $\lambda_{2}=-\left(\alpha_{2}-\delta\right)$ are the eigenvalues of $J_{2}$.

Let us note that $w_{2}=\left(Q(\delta) / d_{1} \alpha_{2}^{2}+\alpha_{2}-\delta ;\left[\beta_{2} d_{2} \delta+\right.\right.$ $\left.\left.\alpha_{2}\left(\alpha_{2}-\delta\right)\right]\left(\alpha_{2}-\delta\right) / \alpha_{2}^{2}\right)$.

The eigenspace associated with $\lambda_{1}$ is $E_{\lambda_{1}}^{(2)}=\left\langle w_{2}\right\rangle$.

The eigenspace associated with $\lambda_{2}$ is $E_{\lambda_{2}}^{(2)}=\langle(0,1)\rangle$. We get the following:

(a) If $Q(\delta)<0$ then $\lambda_{1}<0$ and $\lambda_{2}<0$; then, $U_{2}^{*}$ is stable and its stable manifold is $E^{\mathcal{S}}=\mathbb{R}^{2}$.

(b) If $Q(\delta) \geq 0$ then $\lambda_{1}<0$ and $\lambda_{2} \geq 0$; then $U_{2}^{*}$ is an unstable saddle the unstable manifold of which is $E^{\mathfrak{u}}=\left\langle w_{2}\right\rangle$ and the stable manifold of which is $E^{\mathcal{S}}=\langle(0,1)\rangle$.

(4) Stability of $E^{*}=\left(u^{*} ; v^{*}\right)$ :

Let $\lambda_{1}^{*}$ and $\lambda_{2}^{*}$ be the eigenvalues of $J$. Thus, let us note $\operatorname{Tr}(J)=\lambda_{1}^{*}+\lambda_{2}^{*}$ its trace and $\operatorname{det}(J)=\lambda_{1}^{*} \lambda_{2}^{*}$ its determinant. The eigenspace associated with $\lambda_{1}^{*}$ is $E_{\lambda_{1}}^{*}=\left\langle\left(J_{12} ; \lambda_{1}^{*}-J_{11}\right)\right\rangle$ and the eigenspace associated with $\lambda_{2}^{*}$ is $E_{\lambda_{2}}^{*}=\left\langle\left(J_{21} ; \lambda_{2}^{*}-J_{22}\right)\right\rangle$.

(a) If $\operatorname{det}(J)>0$ and $\operatorname{Tr}(J)<0$ then $\operatorname{Re}\left[\lambda_{1}^{*}\right]<0$ and $\operatorname{Re}\left[\lambda_{2}^{*}\right]<0$. So, $E^{*}$ is stable (stable node or stable focus).

(b) If $\operatorname{det}(J) \geq 0$ and $\operatorname{Tr}(J)=0$ then $\lambda_{1}^{*}, \lambda_{2}^{*} \in i \mathbb{R}$. Therefore $E^{*}$ is a marginal or a center.

(c) If $\operatorname{det}(J)<0$ or $\operatorname{det}(J)>0$ and $\operatorname{Tr}(J)>0$ then $E^{*}$ is unstable. In fact,

(i) if $\operatorname{det}(J)>0$ and $\operatorname{Tr}(J)>0$ then $\operatorname{Re}\left[\lambda_{1}^{*}\right]>0$ and $\operatorname{Re}\left[\lambda_{2}^{*}\right]>0$ or $\lambda_{1}^{*}$ and $\lambda_{2}^{*}$ are conjugated complexes. So, $E^{*}$ is node or an unstable center,

(ii) if $\operatorname{det}(J)<0$ then $E^{*}$ is an unstable saddle.

\subsection{Permanence of Model (9)}

Definition 14 (see [8]). Given solution $u=\left(x_{1}, x_{2}, \ldots, x_{n}\right)$ of a differential system

$$
\dot{u}=f(t, u),
$$

(1) a component $x_{i}$ of the solution $u$ of (39) is said to be weakly persistent if $\lim \sup \left[x_{i}(t)\right]>0$,

(2) a component $x_{i}$ of the solution $u$ of (39) is said to be highly persistent if $\lim \inf \left[x_{i}(t)\right]>0$,
(3) a component $x_{i}$ of the solution $u$ of (39) is said to be uniformly persistent if there is $\varepsilon$ such that $\lim \inf \left[x_{i}(t)\right] \geq \varepsilon>0$,

(4) System (39) is said to be dissipative as for any component $x_{i}$ of the solution there is a constant $M_{i}>0$ such that $\lim \sup \left[x_{i}(t)\right] \geq M_{i}>0$,

(5) System (39) is said to be permanent if it is uniformly persistent and dissipative.

Let $\bar{\Omega}$ be complete metric space and $\Omega$ for an open set such that $\bar{\Omega}=\Omega \cup \partial \Omega$. Further, we shall take $\Omega=\operatorname{int}\left(\mathbb{R}_{+}^{2}\right)$.

Definition 15 (see [8]). A flow or semiflow on $\bar{\Omega}$ under which $\Omega$ and $\partial \Omega$ are forward invariant is said to be permanent if it is dissipative and if there is a number $\varepsilon>0$ such that any trajectory starting in $\Omega$ will be at least at a distance $\varepsilon$ from $\partial \Omega$ for all sufficiently large $t$.

Definition 16 (see [8]). (1) The $\omega$-limit set $\omega(\partial \Omega)$ is said to be isolated if it has a covering $M=\bigcup_{k=1}^{N} M_{k}$ of pairwise disjoint sets $M_{k}$ which are isolated and invariant with respect to the flow or the semiflow both on $\partial \Omega$ and on $\bar{\Omega}=\Omega \cup \partial \Omega$.

(2) The set $\omega(\partial \Omega)$ is said to be acyclic if there exists an isolated covering $M=\bigcup_{k=1}^{N} M_{k}$ such that no subset of $M_{k}$ is a cycle.

Lemma 17 (see [8]). Suppose that a semiflow on $\bar{\Omega}$ leaves both $\Omega$ and $\partial \Omega$ forward invariant, maps bounded sets in $\bar{\Omega}$ to precompact set for $t>0$, and it is dissipative. If in addition

(1) $\omega(\partial \Omega)$ is isolated and acyclic,

(2) $E^{s}\left[M_{k}\right] \cap \Omega=\emptyset$ for all $k \in\{1 ; 2 ; \ldots ; N\}$, where $M=\bigcup_{k=1}^{N} M_{k}$ is the isolated covering used in the definition of acyclicity of $\omega(\partial \Omega)$ and $E^{s}$ denotes the stable manifold,

then the semiflow is permanent.

Theorem 18. Let us assume that $0<\beta_{1}<4$ and $\alpha_{2}>\delta$; then we pose

$$
\begin{aligned}
M_{u}= & \frac{4 d_{2} \beta_{1}+\left(\beta_{1} d_{2}+2 \alpha_{1}\right) \beta_{1} d_{2}+\alpha_{1}^{2}}{\beta_{1} d_{2}\left(4-\beta_{1}\right)} ; \\
L_{1}= & \frac{\left(4+\left(4-\beta_{1}\right) M_{u}\right)^{2}}{16\left(4-\beta_{1}\right)} \\
& +\frac{\left(M_{u}+d_{2}\right)\left(\beta_{2} M_{u}+1+\alpha_{2}-\delta\right)^{2}}{4 \alpha_{2}} ; \\
m_{u}= & -\frac{\beta_{1}}{d_{2}} L_{1}^{2}-\left(\frac{\alpha_{1}}{d_{2}}+\frac{\gamma}{d_{1}}\right) L_{1}+1+\alpha_{1} ; \\
m_{v}= & \frac{\left(\alpha_{2}-\delta\right) d_{2}}{\beta_{2} d_{2}+\alpha_{2}} ;
\end{aligned}
$$




$$
\begin{aligned}
Q(x)= & -d_{2} \beta_{1} d_{1} x^{2} \\
& +\left[\gamma \alpha_{2} d_{2}+d_{2} d_{1} \beta_{1} \alpha_{2}+d_{1} \alpha_{1} \alpha_{2}\right] x \\
& +\alpha_{2}^{2}\left[d_{1}-\gamma d_{2}\right] .
\end{aligned}
$$

Let us consider the following assumptions:

$$
\begin{aligned}
& \left(H_{1}\right) \quad-\frac{\beta_{1}}{d_{2}} L_{1}^{2}-\left(\frac{\alpha_{1}}{d_{2}}+\frac{\gamma}{d_{1}}\right) L_{1}+1+\alpha_{1}>0 ; \\
& \left(H_{2}\right) \quad Q(\delta)>0 .
\end{aligned}
$$

Under the assumptions $\left(H_{1}\right)$ and $\left(H_{2}\right)$, model (9) is permanent and any positive solution $(u, v)$ of $(9)$ verifies

$$
\begin{aligned}
& m_{u} \leq u \leq M_{u} ; \\
& m_{v} \leq v \leq L_{1}-m_{u} .
\end{aligned}
$$

Proof. Given $\Omega=\operatorname{int}\left(\mathbb{R}_{+}^{2}\right)$ and $\partial \Omega=\langle(1 ; 0)\rangle \cup\langle(0 ; 1)\rangle$, its frontier, and $\bar{\Omega}=\Omega \cup \partial \Omega$, we know that $\Omega$ and $\partial \Omega$ are invariants for model (9) (cf. Lemma 3 ) and that $\mathscr{A}$ is attractive bounded for any trajectory from $\bar{\Omega}=\mathbb{R}_{+}^{2}$ (cf. Theorem 6). Let us assume that $0 \leq \beta_{1}<4$ and $\alpha_{2}>\delta$ and let us apply Lemma 17:

(1) Let us justify the fact that model (9) is dissipative over $\bar{\Omega}=\mathbb{R}_{+}^{2}$ :

(a) Let us show that $\liminf [u(t)] \geq m_{u}=-\left(\beta_{1} /\right.$ $\left.d_{2}\right) L_{1}^{2}-\left(\alpha_{1} / d_{2}+\gamma / d_{1}\right) L_{1}+1+\alpha_{1}$. Posing $A_{1}=1$ and $B_{1}=1+\alpha_{1}-L_{1}\left(\beta_{1} L_{1}+\alpha_{1}\right) / d_{2}-\gamma L_{1} / d_{1}$, we have $d u / d t \geq\left(B_{1}-A_{1} u\right) u$ and $u(0)>0$. So, from property (14) of Lemma 4 , we have $\liminf [u(t)] \geq m_{u}=-\left(\beta_{1} / d_{2}\right) L_{1}^{2}-\left(\alpha_{1} / d_{2}+\right.$ $\left.\gamma / d_{1}\right) L_{1}+1+\alpha_{1}$.

(b) Let us show that $\lim \inf [v(t)] \geq m_{v}=\left(\alpha_{2}-\right.$ $\delta) d_{2} /\left(\beta_{2} d_{2}+\alpha_{2}\right)$. Posing $B_{3}=\left(\alpha_{2}-\delta\right)$ and $A_{3}=$ $\left(\beta_{2}+\alpha_{2} / d_{2}\right)$, then, we have $d v / d t \geq\left(B_{3}-A_{3} v\right) v$ and $v(0)>0$. We get $\liminf [v(t)] \geq m_{v}=$ $\left(\alpha_{2}-\delta\right) d_{2} /\left(\beta_{2} d_{2}+\alpha_{2}\right)>0$ for $\alpha_{2}>\delta$.

(c) We deduce that model (9) is dissipative over $\bar{\Omega}=$ $\mathbb{R}_{+}^{2}$ as soon as the assumption $\left(H_{1}\right)$ is verified.

(2) Let us prove that $\omega(\partial \Omega)$ is isolated and acyclic. We have $\partial \Omega=\langle(1 ; 0)\rangle \cup\langle(0 ; 1)\rangle$. On the one hand, $\omega[\langle(1 ; 0)\rangle]=\left\{U_{0}^{*} ; U_{1}^{*}\right\} \subset\langle(1 ; 0)\rangle$; now the stable manifold $U_{1}^{*}$ is $E^{\mathcal{S}}\left[U_{1}^{*}\right]=\langle(1,0)\rangle$ if $\alpha_{2}+\left(1+\alpha_{1}\right) \beta_{2}>\delta$ and $U_{0}^{*}$ is unstable if $\alpha_{2} \geq \delta$. Then any trajectory from $\langle(1 ; 0)\rangle$ other than $U_{0}^{*}$ approaches $U_{1}^{*}$ if $\alpha_{2} \geq \delta$ for $\alpha_{2} \geq \delta \Rightarrow \alpha_{2}+\left(1+\alpha_{1}\right) \beta_{2}>\delta$. On the other hand, $\omega[\langle(0 ; 1)\rangle]=\left\{U_{0} ; U_{2}\right\} \subset\langle(0 ; 1)\rangle$; now the stable manifold of $U_{2}^{*}$ is $E^{s}\left[U_{2}^{*}\right]=\langle(0,1)\rangle$ if $Q(\delta)>0$ and $U_{0}^{*}$ is unstable if $\alpha_{2} \geq \delta$; then any trajectory from $\langle(0 ; 1)\rangle$ other than $U_{0}^{*}$ approaches $U_{2}^{*}$ if $\alpha_{2} \geq \delta$ and $Q(\delta)>0$. Given that $\alpha_{2}>\delta$ then we deduce that $\omega[\partial \Omega]=\left\{U_{0} ; U_{1} ; U_{2}\right\} \subset \partial \Omega$ is isolated and acyclic if $\mathrm{Q}(\delta)>0$ (if the assumption $\left(H_{2}\right)$ is verified).
(3) Let us justify that $E^{s}[\omega(\partial \Omega)] \cap \Omega=\emptyset$. We have $\omega[\partial \Omega]=\left\{U_{0} ; U_{1} ; U_{2}\right\}$ and $\Omega=\operatorname{int}\left(\mathbb{R}_{+}^{2}\right)$. If $\alpha_{2} \geq \delta$, $\alpha_{2}+\left(1+\alpha_{1}\right) \beta_{2}>\delta$, and $Q(\delta)>0$ then $E^{s}[\omega(\partial \Omega)] \cap \Omega=$ $\emptyset$; now $\alpha_{2}>\delta$. So $E^{s}[\omega(\partial \Omega)] \cap \Omega=\emptyset$ if $Q(\delta)>0$.

Definitively, system (9) is permanent.

5.3. Limit Cycle of Model (9). The theorem below presents the conditions for a cyclic growth of the product and the stock of capital of the economy.

Theorem 19. Let us recall the notations of Theorem 13. Let us suppose that the assumptions of Theorem 18 are verified and that model (9) admits a unique interior equilibrium $E^{*}=$ $\left(u^{*} ; v^{*}\right)$. If $\operatorname{det}(J)>0$ and $\operatorname{Tr}(J)>0$ then model (9) admits a limit cycle contained in the attractive region $\mathscr{A}$.

Proof. Under the assumption of Theorem 18, model (9) is permanent and if $\operatorname{det}(J)>0$ and $\operatorname{Tr}(J)>0$ then the unique interior equilibrium $E^{*}=\left(u^{*} ; v^{*}\right)$ is unstable so model (9) admits a limit cycle contained in the compact and bounded region $\mathscr{A}$ (from Poincaré-Beddicton's theorem).

\section{Global Stability of Model (9)}

We now define the conditions in which stability of the product and the stock of capital of the economy are global; that is, they do not depend on the quantities produced and the level of the stock at the initial period. For this study, we define appropriate Lyapunov function.

Theorem 20. Posing $M_{u}=\left(4 d_{2} \beta_{1}+\left(\beta_{1} d_{2}+2 \alpha_{1}\right) \beta_{1} d_{2}+\right.$ $\left.\alpha_{1}^{2}\right) / \beta_{1} d_{2}\left(4-\beta_{1}\right)$ and $L_{1}=\left(4+\left(4-\beta_{1}\right) M_{u}\right)^{2} / 16\left(4-\beta_{1}\right)+$ $\left(M_{u}+d_{2}\right)\left(\beta_{2} M_{u}+1+\alpha_{2}-\delta\right)^{2} / 4 \alpha_{2}$, let us consider the following assumptions:

$$
\begin{aligned}
& 0<\beta_{1}<4 \\
& \alpha_{2}>\delta
\end{aligned}
$$

(9) admits a single point of interior equilibrium $E^{*}$

$$
\begin{aligned}
& =\left(u^{*} ; v^{*}\right), \\
& \frac{\beta_{1} L_{1}^{2}+\alpha_{1} L_{1}}{d_{2}^{2}}+\frac{\gamma L_{1}}{d_{1}^{2}}<1 . \\
& \quad 0<\beta_{1}<\frac{\alpha_{1}}{M_{u}+d_{2}}+\frac{\gamma}{M_{u}+d_{1}} .
\end{aligned}
$$

Under assumptions (43)-(46), the unique interior equilibrium of model (9) is globally and asymptotically stable.

Proof. Let us consider system (9). Let us suppose that assumption (44) is verified; then, model (9) admits a unique interior equilibrium $E^{*}=\left(x^{*}, y^{*}\right)$. 
Let us note that $\lambda=\beta_{1}\left(u+d_{2}\right) / 2\left(\beta_{2} u+\alpha_{2}\right)$. We have $V_{1}: \mathbb{R}^{2} \rightarrow \mathbb{R}$ and $V_{2}: \mathbb{R}^{2} \rightarrow \mathbb{R}$ such that

$$
\begin{aligned}
V_{1}(u, v) & =\left[u-u^{*}-\ln \left(\frac{u}{u^{*}}\right)\right]=\int_{u^{*}}^{u}\left[1-\frac{u^{*}}{x}\right] d x, \\
V_{2}(u, v) & =\lambda\left[v-v^{*}-\ln \left(\frac{v}{v^{*}}\right)\right] \\
& =\lambda \int_{v^{*}}^{v}\left[1-\frac{v^{*}}{x}\right] d x .
\end{aligned}
$$

We have the Lyapunov function $V: \mathbb{R}^{2} \rightarrow \mathbb{R}$ such that $V(u, v)=V_{1}(u, v)+V_{2}(u, v)$.

Then, $d V / d t=d V_{1} / d t+d V_{2} / d t=\left(d V_{1} / d u\right) \dot{u}+$ $\left(d V_{2} / d v\right) \dot{v}=\left(u-u^{*}\right)(\dot{u} / u)+\lambda\left(v-v^{*}\right)(\dot{v} / v)$. Now

$$
\begin{aligned}
& \frac{\dot{u}}{u}=\left[1-u+\left(\beta_{1} v+\alpha_{1}\right)-\frac{v\left(\beta_{1} v+\alpha_{1}\right)}{u+d_{2}}-\frac{\gamma v}{u+d_{1}}\right], \\
& \frac{\dot{v}}{v}=\left[\left(\beta_{2} u+\alpha_{2}-\delta\right)-\frac{\left(\beta_{2} u+\alpha_{2}\right)}{u+d_{2}} v\right],
\end{aligned}
$$

and $E^{*}=\left(u^{*} ; v^{*}\right) \in\left(\mathbb{R}_{+}^{*}\right)^{2}$, a unique interior equilibrium of (9); then,

$$
\begin{aligned}
& 1=u^{*}-\left(\beta_{1} v^{*}+\alpha_{1}\right)\left(1-\frac{v^{*}}{u^{*}+d_{2}}\right)+\frac{\gamma v^{*}}{u^{*}+d_{1}} ; \\
& \alpha_{2}-\delta=-\beta_{2} u^{*}+\frac{\left(\beta_{2} u^{*}+\alpha_{2}\right) v^{*}}{u^{*}+d_{2}} .
\end{aligned}
$$

Therefore,

$$
\begin{aligned}
\frac{\dot{u}}{u} & =\left(u-u^{*}\right)\left[-1+\frac{\beta_{1} v^{2}+\alpha_{1} v}{\left(u^{*}+d_{2}\right)\left(u+d_{2}\right)}\right. \\
& \left.+\frac{\gamma v}{\left(u^{*}+d_{1}\right)\left(u+d_{1}\right)}\right]+\left(v-v^{*}\right)\left[\beta_{1}\right. \\
& \left.-\frac{\beta_{1}\left(v+v^{*}\right)+\alpha_{1}}{\left(u^{*}+d_{2}\right)}-\frac{\gamma}{\left(u^{*}+d_{1}\right)}\right] ; \\
\frac{\dot{v}}{v} & =\left(u-u^{*}\right)\left[-\beta_{2}-\frac{\beta_{2} v^{*}}{u^{*}+d_{2}}+\frac{\left(\beta_{2} u+\alpha_{2}\right) v}{\left(u^{*}+d_{2}\right)\left(u+d_{2}\right)}\right] \\
& -\frac{\left(\beta_{2} u+\alpha_{2}\right)}{u^{*}+d_{2}}\left(v-v^{*}\right) .
\end{aligned}
$$

Let us pose the following:

$$
\begin{aligned}
& g(u, v) \\
& =-1+\frac{\beta_{1} v^{2}+\alpha_{1} v}{\left(u^{*}+d_{2}\right)\left(u+d_{2}\right)}+\frac{\gamma v}{\left(u^{*}+d_{1}\right)\left(u+d_{1}\right)} ; \\
& h(u, v) \\
& =\frac{1}{2}\left[\beta_{1}-\frac{\beta_{1}\left(v+v^{*}\right)+\alpha_{1}}{\left(u^{*}+d_{2}\right)}-\frac{\gamma}{\left(u^{*}+d_{1}\right)}\right] \\
& \quad+\frac{\lambda}{2}\left[-\beta_{2}-\frac{\beta_{2} v^{*}}{u^{*}+d_{2}}+\frac{\left(\beta_{2} u+\alpha_{2}\right) v}{\left(u^{*}+d_{2}\right)\left(u+d_{2}\right)}\right] .
\end{aligned}
$$

Consequently, $d V / d t=g(u, v)\left(u-u^{*}\right)^{2}+2 h(u, v)\left(u-u^{*}\right)(v-$ $\left.v^{*}\right)-\lambda\left(\left(\beta_{2} u+\alpha_{2}\right) /\left(u^{*}+d_{2}\right)\right)\left(v-v^{*}\right)^{2}$. Then, $d V / d t \leq[g(u, v)+$ $h(u, v)]\left(u-u^{*}\right)^{2}+\left[h(u, v)-\lambda\left(\left(\beta_{2} u+\alpha_{2}\right) /\left(u^{*}+d_{2}\right)\right)\right]\left(v-v^{*}\right)^{2}$. Thus, $d V / d t<0$ if $g(u, v)<0$ and $h(u, v)<0, \forall(u, v) \in \mathbb{R}^{2}$.

Let us determine the conditions on the control parameters of model (9) such that $g(u, v)<0$ and $h(u, v)<0$, $\forall(u, v) \in \mathbb{R}^{2}$ (cf. [9], pages 110-111).

(1) Let us overestimate $g(u, v)$. We have $g(u, v)=-1+$ $\left(\beta_{1} v^{2}+\alpha_{1} v\right) /\left(u^{*}+d_{2}\right)\left(u+d_{2}\right)+\gamma v /\left(u^{*}+d_{1}\right)(u+$ $\left.d_{1}\right)$. Then $g(u, v) \leq-1+\left(\beta_{1} L_{1}^{2}+\alpha_{1} L_{1}\right) / d_{2}^{2}+\gamma L_{1} / d_{1}^{2}$. Therefore $g(u, v)<0, \forall(u, v) \in \mathbb{R}^{2}$ if $-1+\left(\beta_{1} L_{1}^{2}+\right.$ $\left.\alpha_{1} L_{1}\right) / d_{2}^{2}+\gamma L_{1} / d_{1}^{2}<0$.

(2) Let us overestimate $h(u, v)$. We have $\partial h / \partial v=(-(u+$ $\left.\left.d_{2}\right) \beta_{1}+\lambda\left(\beta_{2} u+\alpha_{2}\right)\right) / 2\left(u^{*}+d_{2}\right)\left(u+d_{2}\right)$. Now $\lambda=$ $\beta_{1}\left(u+d_{2}\right) / 2\left(\beta_{2} u+\alpha_{2}\right)$; then, $\partial h / \partial v=-\beta_{1} / 4\left(u^{*}+d_{2}\right)<$ 0 . Consequently, $h(u, v) \leq h(u, 0), \forall v \geq 0$. Then, $h(u, v) \leq(1 / 2)\left[\beta_{1}-\alpha_{1} /\left(u^{*}+d_{2}\right)-\gamma /\left(u^{*}+d_{1}\right)\right]+$ $(\lambda / 2)\left[-\beta_{2}-\beta_{2} v^{*} /\left(u^{*}+d_{2}\right)\right]$. Therefore, $h(u, v) \leq 0$, $\forall(u, v) \in \mathbb{R}^{2}$, if $\beta_{1}<\alpha_{1} /\left(M_{u}+d_{2}\right)+\gamma /\left(M_{u}+d_{1}\right)$.

(3) Let us deduce that $d V / d t<0$. We know that $\left(\beta_{1} L_{1}^{2}+\right.$ $\left.\alpha_{1} L_{1}\right) / d_{2}^{2}+\gamma L_{1} / d_{1}^{2}<1 \Rightarrow g(u, v)<0, \forall(u, v) \in \mathbb{R}^{2}$, and $\beta_{1}<\alpha_{1} /\left(M_{u}+d_{2}\right)+\gamma /\left(M_{u}+d_{1}\right) \Rightarrow h(u, v) \leq 0$, $\forall(u, v) \in \mathbb{R}^{2}$. Now $d V / d t<0, \forall(u, v) \in \mathbb{R}^{2}$, if $g(u, v)<0$ and $h(u, v)<0, \forall(u, v) \in \mathbb{R}^{2}$. Then, $d V / d t<0, \forall(u, v) \in \mathbb{R}^{2}$, if assumptions (43)-(46) are verified. Therefore the unique interior equilibrium of model (9) is globally and asymptotically stable if assumptions (43)-(46) are verified.

\section{Conclusion}

Our work used the Kaldor model as basic economic model. By including it at the level of the investment rate and saving rate compatible ecological functions, we encourage the economic actors to adopt a behaviour permitting very rapidly entering a stability area (attracting set $\mathscr{A}$ ). This stability can be noticed on the one hand in the form of stationary growth of the stock of capital and the product (stable interior equilibrium) and on the other hand in the form of cyclic growth of the capital and the product (limit cycle). We therefore guarantee, under certain conditions, the permanence of the stock of capital, $K$, and that of the product, $Y$, in the economy avoiding, in that way, a shortage of the stock of capital or the production in the long term. Under certain conditions, this stability of the financial system (in relation to the capital and the product) is global; that is, it does depend on the level of the stock of capital and the level of production at the initial period.

In the first consideration, the model can be applied to a state, regional organisation, or to an enterprise. In the case of an enterprise, the product $Y$ refers to the monetary value of the production. We can then infuse the existing production functions such as Cobb-Douglas, Leontief, and CES. In that case, we can substitute the saving with the quantity of work and the tendency will show a technical progress. 
Secondly, the model can also be applied as an ecological model of two species where one of the species (e.g., man) "cultivate" the other species for its survival or to prevent the loss of that species through a culture rate (investment rate) which is nonnull.

\section{Conflicts of Interest}

The authors declare that they have no conflicts of interest.

\section{References}

[1] R. E. Ulanowicz, "The dual nature of ecosystem dynamics," Ecological Modelling, vol. 220, no. 16, pp. 1886-1892, 2009.

[2] A. G. Haldane and R. M. May, "Systemic risk in banking ecosystems," Nature, vol. 469, no. 7330, pp. 351-355, 2011.

[3] H.-W. Lorenz, Nonlinear Dynamical Economics and Chaotic Motion, Springer-Verlag, Berlin, Germany, 2 edition, 1993.

[4] J. Glombowski and M. Krüger, "A Short Periode Growth Model Model," Recherche économique de Louvain, vol. 54, no. 4, 1988.

[5] M. Volle, "Prédation et prédateurs," Economica, vol. 201, january 2008.

[6] F. Chen, X. Liao, and Z. Huang, "The dynamic behavior of $N$ species cooperation system with continuous time delays and feedback controls," Applied Mathematics and Computation, vol. 181, no. 2, pp. 803-815, 2006.

[7] M. A. Aziz Alaoui and M. Daher Okaye, "Boundness and global stability for a predator-prey model with modified Leslie Gower and Holling-type 2," Applied Mathematical Letters, vol. 16, pp. 1069-1075, 2003.

[8] W. Khellaf and N. Hamri, "Boundedness and global stability for a predator-prey system with the beddington-deangelis functional response," Differential Equations and Nonlinear Mechanics, vol. 2010, Article ID 813289, p. 24, 2010.

[9] A. F. Nindjin, M. A. Aziz-Alaoui, and M. Cadivel, "Analysis of predator-prey model with modified leslie-gower and hollingtype II schemes with time delay," Nonlinear Analysis: Real World Applications, vol. 7, no. 5, pp. 1104-1118, 2006. 


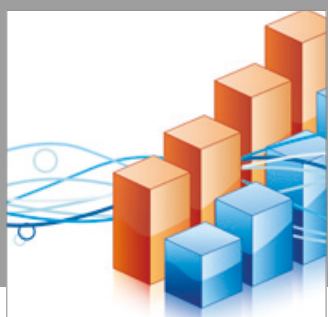

Advances in

Operations Research

vatersals

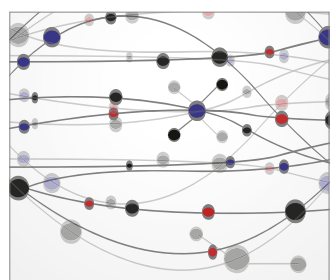

\section{The Scientific} World Journal
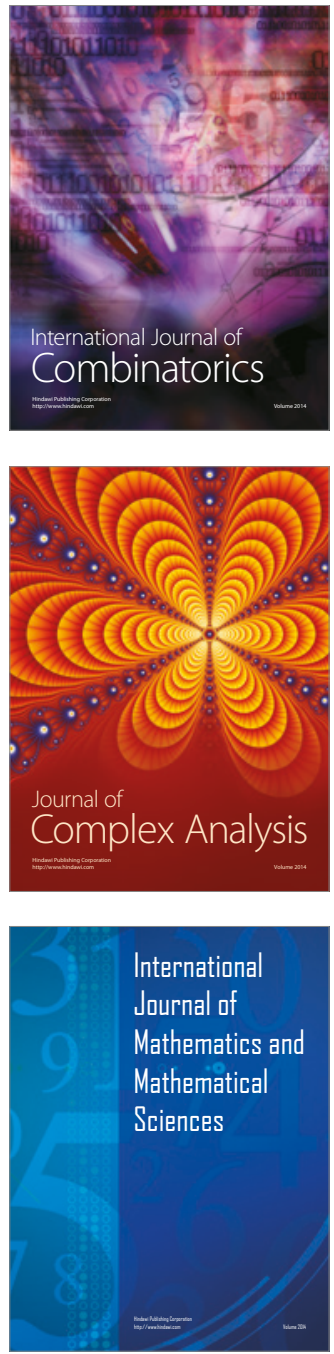
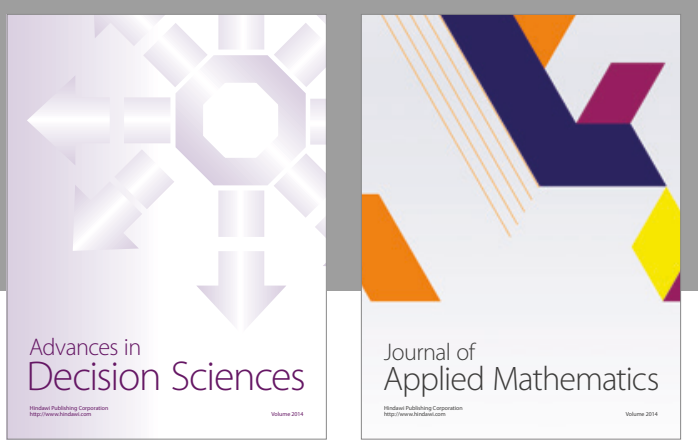

Algebra

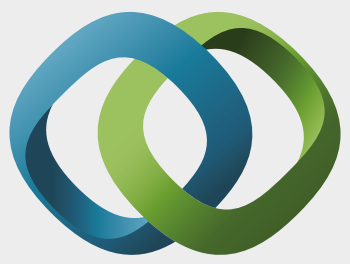

\section{Hindawi}

Submit your manuscripts at

https://www.hindawi.com
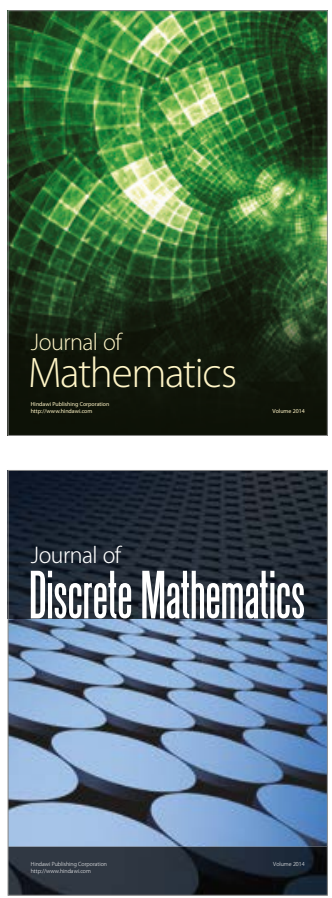

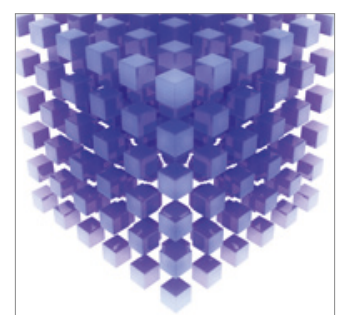

Mathematical Problems in Engineering
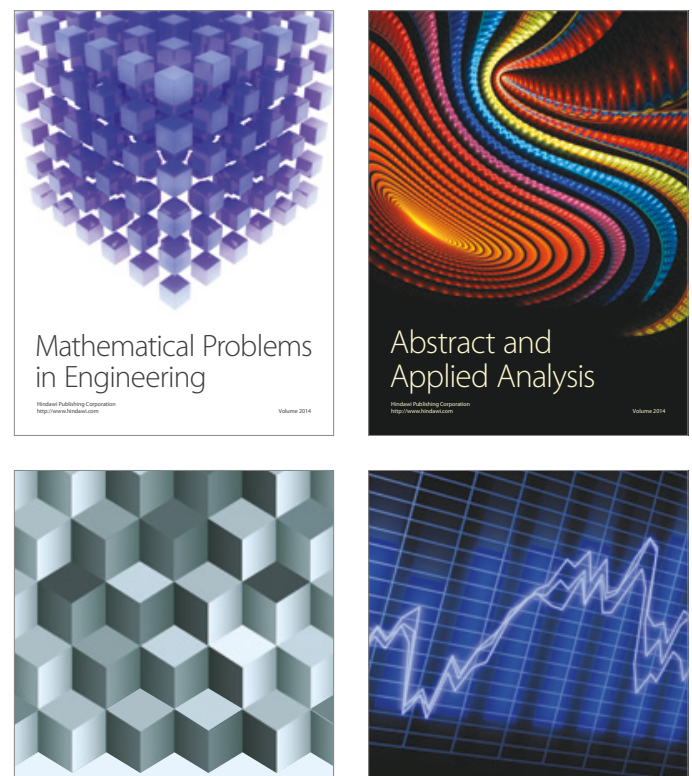

Journal of

Function Spaces

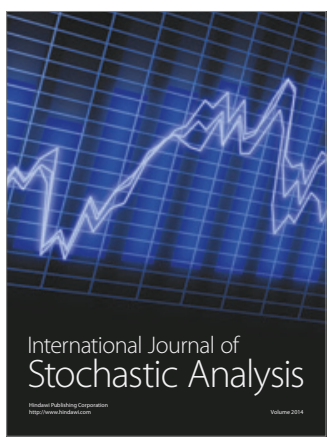

Probability and Statistics
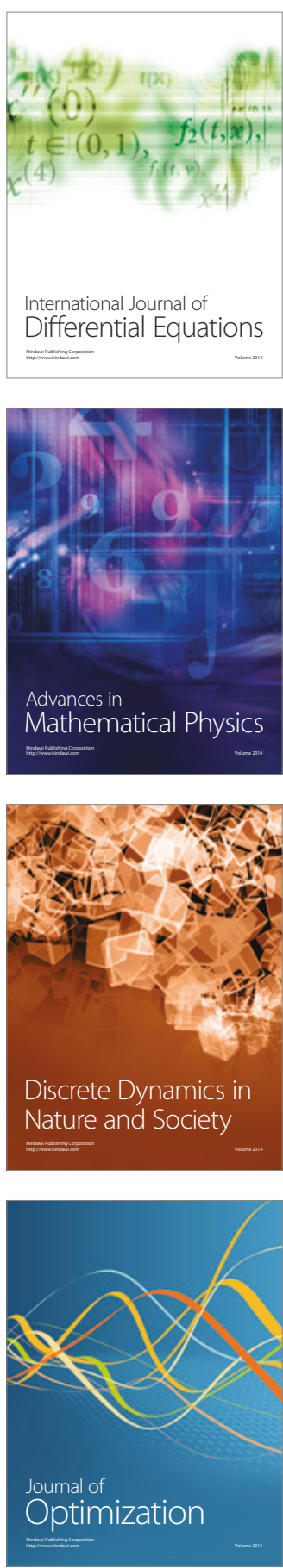